\title{
A Stage 1 Pilot Cohort Exploring the Use of EMDR Therapy as a Video-Conference Psychotherapy During Covid-19 - A Proof of Concept Study Utilising a Virtual Blind 2 Therapist Protocol
}

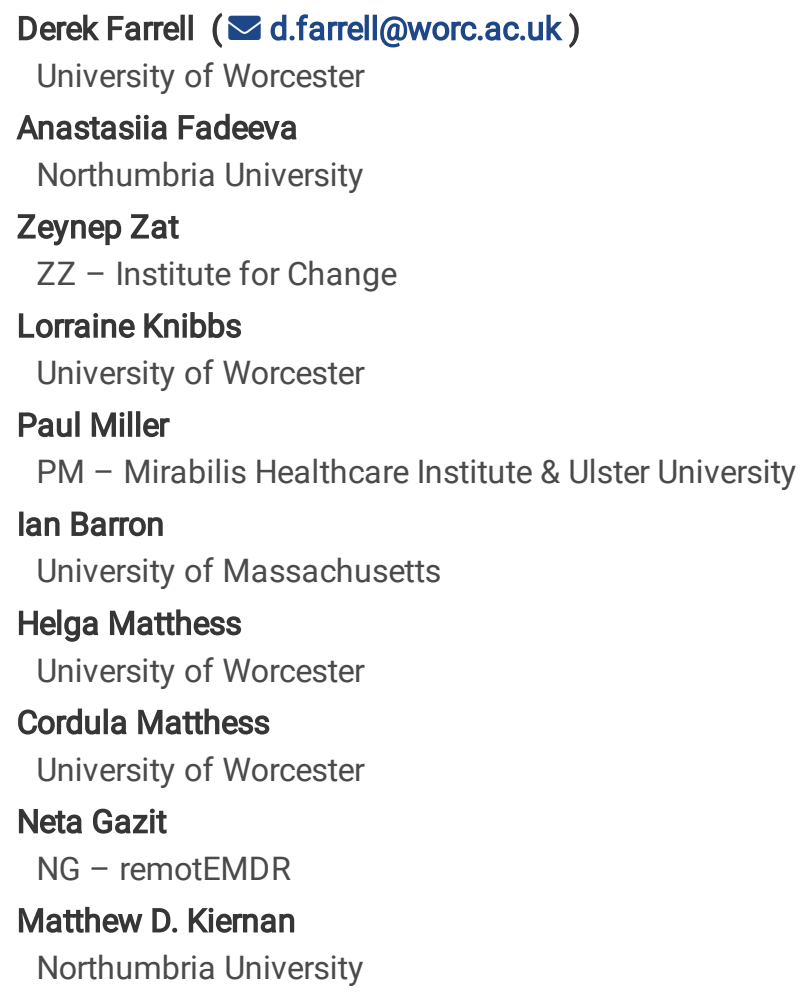

\section{Research Article}

Keywords: EMDR therapy, pathogenic memory, adverse and benevolent childhood experiences, video-conference psychotherapy, blind 2 therapist.

Posted Date: October 20th, 2021

DOI: https://doi.org/10.21203/rs.3.rs-886940/v1

License: 두 (i) This work is licensed under a Creative Commons Attribution 4.0 International License. Read Full License 


\section{Abstract \\ Background}

The Covid-19 pandemic has had a major impact in the delivery of psychological treatment. Due to social distancing requirements provision moved to video-conferencing psychotherapy (VCP). There is a paucity of empirical data supporting the efficacy of EMDR therapy as a VCP. This stage 1 pilot study tested an EMDR therapy scripted protocol - Virtual Blind 2 Therapist (VB2T) as a VCP regarding fitness for purpose, distinctiveness, relevance, and efficiency.

\section{Methods}

Twenty-four participants were recruited to the study. The design included a one-session treatment intervention obtaining measures pre, post, one-month, and six-month follow-up. This treatment session used a 'Blind 2 Therapist' EMDR therapy scripted protocol as videoconference psychotherapy that involves non-disclosure of traumatic memory. The research explored the treatment effect on the core characteristics of the trauma memory including subjective disturbance, belief systems, memory intensity, vividness, and levels of emotionality. Additionally, the research explored participant's experiences of adverse and benevolent childhood experiences (ACEs/BCEs) during their childhood.

\section{Results}

Regarding the four tests - fitness for purpose, distinctiveness, relevance, and efficiency results are favourably suggesting potential clinical benefits of using EMDR as video-conference psychotherapy. That said this is a proof-of-concept study showing positive results, however, no clinical population or control group was used. The purpose of the study is to explore the potential for scalability towards a larger clinical trial. The treatment intervention was achieved irrespective of either adverse or benevolent childhood experiences (ACEs/BCEs) during childhood

\section{Conclusion}

The research tentatively supports the case for EMDR therapy as a credible treatment when used as a video conference psychotherapy. More research is needed to scale towards a clinical trial.

\section{Background}

In the attempt to reduce the risk of infections from Covid-19, many mental health care providers are closing their doors to patients requiring face-to-face therapy and instead of creating video-conferencing psychotherapy (VCP), remote access technology, e-health tools, and internet interventions $(1,2)$. Several comprehensive reviews highlight the effectiveness of video-conferencing psychotherapy (VCP) and therapist-guided interventions, with conditions such as anxiety, major depressive disorders, and trauma (1-15). VCP appear both 'feasible' and 'acceptable' as a mode of psychological treatment delivery, providing high satisfaction and effectiveness (16). Furthermore, VCP provides a viable alternative in providing continuity of care in times of social, economic, and health upheaval (17).

There are several distinct barriers towards the equitable provision of, and access to, evidence-based, face to face/in-person psychological treatments with a paucity of suitably qualified mental health workers to sufficiently address the global burden of mental illness and psychological trauma (18). With relatively few providers trained in the therapies underpinned by a solid empirical evidence base, those who live in rural or remote communities are further restricted. The barriers to care compounded further by disability, poverty, and stigma $(13,19,20)$. VCP provides alternative flexibility and equity of access than in-person therapy, with potential for financial efficiencies and cost savings, enhanced reach, flexible implementation, improved cultural adaptability and sensitivity, and improved equity when compared with in-person therapy $(19,21-25)$.

Despite potential benefits, VCP does require critical consideration. One factor relates to the impact VCP has on the therapeutic alliance when assuming the 'In person', traditional model, the gold standard for psychotherapy. Recent studies $(6,16,26)$ acknowledge that an effective therapeutic alliance is essential in underlying successful therapy. However, Simpsom (16). Table 1 outlines the advantages and disadvantages of using VCP's and how they can be adapted to promote greater effectiveness.

Page $2 / 22$ 
Table 1

Advantages, disadvantages, and adaption factors in VCP's

\begin{tabular}{|c|c|c|}
\hline Advantages & Disadvantages & Adaptation Factors to promote greater effectiveness \\
\hline $\begin{array}{l}\text { o Greater flexibility } \\
\text { o Cultural adaptability } \\
\text { o Enhanced reach } \\
\text { o Better use of scarce } \\
\text { resources } \\
\text { o Cost efficiencies } \\
\text { o Increased accessibility } \\
\text { o No geographical } \\
\text { restrictions } \\
\text { o Environmentally - } \\
\text { reduces carbon } \\
\text { footprint } \\
\text { o Responds to the need } \\
\text { for rural services for } \\
\text { veterans } \\
\text { o Convenience and } \\
\text { affordability for } \\
\text { disabled people }\end{array}$ & $\begin{array}{l}\text { o Technology knowledge, application, } \\
\text { functioning, and reliability, including } \\
\text { challenges } \\
\text { o Poor internet connections, particularly } \\
\text { in low socioeconomic areas } \\
\text { o Body language restricted to head and } \\
\text { face } \\
\text { o Privacy into the home environment } \\
\text { o Creating safe space, time, and } \\
\text { relationships } \\
\text { o Cultural considerations and norms } \\
\text { o Risk management \& triage } \\
\text { o Geographical factors, legislation, } \\
\text { professional indemnity, logistics } \\
\text { o Insurance cover and liability }\end{array}$ & $\begin{array}{l}\text { o Adjusting for more restricted access to non-verbal } \\
\text { communication } \\
\text { o More regular 'checking in' with clients } \\
\text { o Requesting more information and clarification on specific } \\
\text { points } \\
\text { o More focus on facial expressions and bodily gestures } \\
\text { o More frequent emotion checking } \\
\text { o Enhanced preparation before sessions } \\
\text { o More control of the space } \\
\text { o The therapists assuming a greater sense of ownership } \\
\text { and responsibility for the therapeutic alliance within the } \\
\text { therapy work } \\
\text { o Client centredness - the Therapist 'tailoring' their } \\
\text { approach more to the client individual and specific needs }\end{array}$ \\
\hline
\end{tabular}

The reality of the Covid-19 pandemic ostensibly has removed choice for a great many individuals, the options being VCP intervention, no intervention, or an extensive and uncertain period of waiting. The current advances in VCP technology enable it to offer an innovative solution as a viable alternative to in-person therapies.

The World Health Organisation(27) has expressed concern over the psychological impact that the pandemic and social distancing has had on the mental health of a broad sector of society. The psychosocial consequences include increases in loneliness, anxiety, depression, gender-based violence, insomnia, substance misuse, self-injurious activity, and suicidal behaviour (28-34). However, Covid19 is not 'ground zero' when considering the mental wellbeing of a population. Those with pre-existing mental health issues before the pandemic risk minimisation, reclassification, and systemic loss within existing health care systems. It is essential to acknowledge that events that pre-date Covid-19 may still influence an individual's response. Two considerations are exposure to Adverse Childhood Experiences (ACEs) and Benevolent Childhood Experiences (BCEs). Exposure to ACEs is the single most potent global public health issue when considering social inequality, lifelong impact on health and behaviour, and social deprivation. Arguably the Covid-19 pandemic further compounds antecedent ACEs. Social inequalities such as these create significant barriers when accessing services, either in person or via VCP (35-44).

VCP interventions have primarily focussed upon Prolonged Exposure (13,45-50), Behavioural Activation (45, 46, 51), Cognitive Processing Therapy $(19,52-54)$ and CBT $(55,56)$. Presently, limited research publications support EMDR therapy which uses VCP to treat Post-Traumatic Stress Disorder (PTSD (57-59). However, a recent study (60) used home-based psychotherapy, delivered by telehealth, as a treatment intervention for complex PTSD. The intervention was delivered in an intensive format, offering both Prolonged Exposure and EMDR therapy. Six participants took part in the study, with two-thirds losing their PTSD or complex-PTSD diagnostic status the study demonstrating that the telehealth intervention was both safe and effective (60). However, the sample size in their study was small, with just six participants, with only four of the six losing their diagnosis. Safety and effectiveness determinants of the EMDR therapy intervention indicate more extensive and more representative sample sizes.

EMDR therapy, an empirically supported intervention for PTSD and Complex PTSD $(7,61-66)$ was developed in the late 1980 s by an American psychologist - Francine Shapiro. Its primary foci are on the treatment of pathogenic memories and their associated stress symptoms with the model of pathogenesis and change known as Adaptative Information Processing (67, 68). Shapiro (69) considered trauma memories in a range of mental health disorders and not just PTSD and Complex PTSD. Within the AIP theoretical framework, a meta-theory unique to EMDR therapy, the model assumes that the human brain can usually process memories of adverse life events to complete integration. The essence of EMDR therapy involves four distinct aspects - preparation, access, stimulation, and integration [PACI]. What gives EMDR therapy a specific distinctness relates to bi-focal physical stimulation, a working memory taxation device that 
enables the client to attend internal and external stimuli $(66,70,71)$. The hypothesised working mechanism of EMDR therapy is still under investigation (72). However, most evidence supports the working memory account. Working memory has a limited capacity. Therefore, dual taxation sets up a competing situation. Consequently, the emotional intensity of the pathogenic memory, with all its subjective levels of disturbance, is gradually lost and eventually reconsolidated into a less disturbing and reduced emotional form. Within the EMDR therapy literature, the dominant empirical evidence supports physical eye movements; however, other forms of bi-focal physical stimulation can include acoustic, somatic, or multiple forms such as is used within EMDR 2.0 (71).

The core characteristics of the EMDR B2T protocol access and activate a pathogenic memory. However, the primary distinction from the Standard Protocol is that the client reveals no details about the memory itself, other than its emotional and somatic content, and an indication of their subjective unit of distress (SUD). Clients are under no pressure to disclose any of the trauma content during trauma processing using B2T. Table 2 highlights the core components of the EMDR therapy B2T, its context regarding the eight phases of therapy, and the assessment phase (Phase 3 of the standard protocol of EMDR therapy).

Table 2

EMDR Blind 2 Therapist Protocol in Context - adapted from Farrell et al., (18)

\begin{tabular}{|lll|}
\hline $\begin{array}{l}\text { EMDR therapy: } 8 \\
\text { phases }\end{array}$ & $\begin{array}{l}\text { EMDR therapy Phase 3 Standard Protocol - } \\
\text { Assessment Structure }\end{array}$ & $\begin{array}{l}\text { EMDR therapy Phase } 3 \text { Assessment Blind 2 Therapist } \\
\text { structure and VB2T version }\end{array}$ \\
\hline $\begin{array}{l}\text { Phase 1: history } \\
\text { taking }\end{array}$ & Target Memory & Target memory - cue word \\
$\begin{array}{l}\text { Phase 2: } \\
\text { preparation }\end{array}$ & Worst Image & Emotions \\
$\begin{array}{l}\text { Phase 3: } \\
\text { assessment }\end{array}$ & Positive Cognition & Subjective Unit of disturbance (SUD 0-10) \\
$\begin{array}{l}\text { Phase 4: } \\
\text { desensitisation }\end{array}$ & Validity of Cognition (1-7) & Location of body sensation \\
$\begin{array}{l}\text { Phase 5: } \\
\text { installation }\end{array}$ & Subjective Unit of Disturbance (0-10) & \\
$\begin{array}{l}\text { Phase 6: body } \\
\text { scan }\end{array}$ & Location of body sensation & \\
$\begin{array}{l}\text { Phase 7: closure } \\
\begin{array}{l}\text { Phase 8: re- } \\
\text { evaluation }\end{array}\end{array}$ & \\
\hline
\end{tabular}

Many empirically supported treatments for PTSD contain various elements and degrees of exposure. These rely upon the client's ability and willingness to disclose the memory of the adverse life event causing a stress response. When pathogenic memories involve shame, guilt; disgust; fear of retribution; lack of language; non-disclosure self-protection/ preservation factors, then disclosure may not even be a viable option for a client. A study published from research carried out in Northern Iraq tested an adaption of EMDR therapy known as the 'Blind 2 Therapist' (B2T) protocol. This study demonstrated the safety, effectiveness, efficiency and relevance as a treatment intervention for both 'shame-based and' fear of retribution' trauma (18). However, an evaluation of EMDR B2T as a VCP method of delivery has not occurred to date. Therefore, this study aimed to test the virtual version of B2T (referred to as VB2T), delivered as a VCP, as a suitable clinical intervention in the desensitisation and reprocessing of a pathogenic memory.

As indicated earlier, within the existing EMDR therapy literature, there is a paucity of research into the use of VCP EMDR (60). In addressing this aspect any potential study would have to address two significant aims:

1. What adaptations, if any, would be required to use EMDR therapy as a VCP?

2. Critically consider potential advantages/ disadvantages of EMDR therapy as a VCP?

Therefore, the purpose of this study was to determine whether the application of VCP EMDR therapy would be associated with (1) Fitness for purpose - safe and effective, (2) Distinctiveness - alterations of the core components of a pathogenic memory regarding intensity, vividness, and emotionality, (3) Relevance, and (4) Efficiency.

A directional hypothesis is a prediction of positive or negative change between two variables in a specific population. These changes were measured by pre, post, 1-month, and 6-month follow-up. The research study hypotheses were as follows: 
Hypothesis 1 Fitness for Purpose - Virtual Blind 2 Therapist EMDR (VB2T), when delivered as a video-conference psychotherapy, will have no impact on the Subjective Unit of Disturbance (SUD) and the Validity of Cognition (VOC) in regard to a pathogenic memory when measured at post-treatment, 1-mth and 6-mth in comparison with a pre-measure.

Hypothesis 2 Distinctiveness - VB2T, as a VCP, will have no impact in reducing Memory Intensity (MI), Memory Emotionality (ME), and Memory Vividness (MV) of a pathogenic memory following intervention when measured at post-treatment, 1-mth and 6-mth in comparison with a pre-measure.

Hypothesis 3 Relevance -When using VB2T, Adverse Childhood Experiences (ACEs) or Benevolent Childhood Experiences (BCEs) will influence the processing of a pathogenic memory.

Hypothesis 4 Efficiency - VB2T will take longer in time than the 60-90 minutes recommended by www.emdria.org, and therefore would be more expensive as a clinical intervention.

\section{Methodology - Research Design}

\section{Ethical approval}

for the study was granted through University of Worcester (UK) [CBPS1920031-R2]. Consequently, all the methods used for the study were carried out in strict adherence to the ethical approval granted and in accordance with relevant guidelines and regulations. Informed consent was obtained from all subjects. Additionally, the study was registered as a clinical trial ID ICSRCTN12099530 [30/06/2021]. This stage 1 pilot study used a pre-test/post-test design taking measures before and after a one-session treatment using the EMDR VB2T protocol, including 1-month and 6-month follow-up to determine the impact of the treatment intervention on the pilot cohort. The rationale for an experimental design as a stage 1 research project was to determine proof of concept before proceeding to stage 2 involving a quasi-experimental design utilising a distinct control group. The longer-term strategy is for phases 1 and 2 to support a more significant funding application utilising a randomised control design incorporating a delayed treatment paradigm.

\section{Participants}

Using EMDR therapy and the VB2T protocol as a VCP is a contemporaneous research idea in relation to the software package needed to deliver it. Mindful of this the VCP software developers wanted to test their product to be safe as an application for clinical practice, more particularly with EMDR therapy. Consequently, the study used a within-community population group consisting of highly experienced, UK based, EMDR therapists. The design of the study incorporated a self-selecting (volunteer) sampling. The inclusion criteria were: -

- EMDR Europe Accredited Consultant,

- Encountered an adverse life event that generated a presently held, subjective level of disturbance (SUD)

- Willingness to be a client for a one-session intervention using the EMDR therapy Blind 2 Therapists protocol (VB2T) as a VCP, using the remotEMDR platform.

This sampling method was selected as it is highly consistent with what occurs during accredited EMDR therapy and CBT teaching and learning programmes $(18,73-76)$. A sample size of 17 was deemed sufficient to compare findings from the original study, which used the EMDR Blind 2 therapist protocol with participants from Northern Iraq (18). However, as $\mathrm{N}=24$ met the required inclusion criteria, all were recruited into the study.

\section{Measures Used For The Study}

The pre-test/post-test design utilised the following measures:

Subjective Unit of Disturbance (SUD) is a scale of 0-10 for measuring subjective levels of distress or disturbance currently experienced by an individual (77).

Validity of Cognition Scale (VOC) provides a rapid assessment of cognitive structure on an emotional/ somatic level rather than intellectual $(69,78,79)$. Both the SUD and VOC have documented validity, reliability, and correlations with several physiological indices of distress. 
Memory Vividness (MV) and Emotionality (ME) - Subjective unit of measurement $(0-10)$ of the vividness of the target memory, either positive or negative (80-87).

Memory Intensity $(\mathrm{MI})$ - Subjective unit of measurement $(0-10)$ of the intensity of the target memory, either positive or negative (8890).

Adverse Childhood Experiences Scale (ACE) collect crucial information based upon the prevalence of adversity during childhood in ten categories before the age of eighteen: emotional abuse (recurrent), physical abuse (recurrent), sexual abuse (contact), physical neglect, emotional neglect, substance misuse in the household, mental illness in the household, mother treated violently, divorce or parental separation, and criminal behaviour in the household (41). With each category counting as one point, with ten categories, the highest possible ACE score is $10(40,42,91-96)$. An additional component of the ACEs was to test the study participants group for comparability with the original CDC Kaiser Permanente Study (42). Because it is a UK study, the ACEs replication studied carried out Bellis et al., 2015).

Benevolent Childhood Experiences (BCEs) scale is a new instrument designed to assess positive early life experiences in adults with a history of childhood adverse experiences. Ostensibly, BCEs, are a counterpart to the Adverse Childhood Experiences (ACEs) questionnaire. The BCEs (95) are multiculturally sensitive and applicable regardless of socioeconomic position, urban-rural background, or immigration status. The BCEs items utilise developmental psychology framework, integrated with ecological systems theory $(64,95$, 97-103).

Time (minutes) using the metric period recommended by EMDRIA sessions; $60-90$ minutes, measuring from commencement of Phase 3 - Assessment, to completion of Phase 7 - Closure (including debrief).

Cost per session ( $\left.£^{\prime} / €^{\prime} s\right)$ using economic modelling from the University of Worcester calculated at $£ 56.49$ ( $\left.€ 66.36\right)$.

One month after each VB2T session, another research team member carried out Phase 8 Re-evaluation, conducted a qualitative interview, and obtained 1-month follow-up data-additional psychometric data collected at 6-months also.

\section{Treatment}

The research utilised a 1-treatment session intervention (EMDR VB2T as a VCP), a partial replication from a previous study (18). This study used a beta-tested software programme called remotEMDR (https://www.remotEMDR.com), a technology that enabled the delivery of EMDR as a VCP. The remotEMDR is a synchronous programme that offers various visual and acoustic forms of bi-focal physical stimulation and includes an integrative video platform, giving EMDR therapists complete control within the session.

The EMDR therapy VB2T protocol, adapted for VCP usage, originated from the original B2T (104), including pathogenic memory vividness, intensity, and emotionality metrics.

An EMDR Europe Accredited Senior Trainer and Consultant carried out each of the VB2T treatment sessions, each digitally recorded and made available for treatment fidelity checking. EMDR Europe Consultants and Co-researchers carried out these fidelity checks to the project. The EMDR Foundation Fidelity Rating Scale (EFRS) - version 2 developed initially by der Kolk et al. (90), subsequently revised and updated by Maxfield et al (105).

VB2P sessions incorporated multiple consent points, including initial recruitment, the commencement of the VB2T session, permission to record digitally, and permission to utilise the research participants data at the end of the VB2T session.

\section{Statistical analysis}

Statistical analysis utilised the Statistical Package for Social Sciences (SPSS version 26.0; Chicago, IL, USA) to include means and standard deviations calculated for SUD, VOC, MV, ME, and MI before treatment, post-treatment, and at 1- and 6-months follow-up (Table 3). Data sets included skewness and kurtosis, calculated to estimate the normality of the outcome measures, frequencies of total and individual scores for ACEs and BCEs. This detailed examination and alpha testing, which included generalised estimating equations (GEE) to compare before/after the intervention, and follow-up changes in SUD, VOC, MV, ME, and MI. Added were ACEs and BCEs as the covariates in the modelling exercise. The GEE model accounts for time variations and correlations among repeated measurements and does not require the dependent variable to be normally distributed $(106,107)$. Gamma with log link was selected as the outcome 
variables were skewed. The presence of negative values for SUD, MV, ME, and MI measures was handled by adding a constant value to the data before the analysis. As for descriptive statistics, we used mean \pm standard deviation ( $\pm S D$ ) for numerical variables and percentage (\%) for categoric variables. $p$-Values of $<.05$ were considered significant. An overall effect size using Hedges $g$ was calculated.

Table 3

Descriptives - Subjective Unit of Disturbance (SUD) \& Validity of Cognition (VOC): pre, post, 1-mth, \& 6-mth follow-up

\begin{tabular}{|c|c|c|c|c|c|c|c|c|c|}
\hline & Intervention & $\begin{array}{l}\text { Pre } \\
\text { SUD }\end{array}$ & $\begin{array}{l}\text { Post } \\
\text { SUD }\end{array}$ & $\begin{array}{l}\text { SUD 1- } \\
\text { mth FU }\end{array}$ & $\begin{array}{l}\text { SUD 6- } \\
\text { mth FU }\end{array}$ & $\begin{array}{l}\text { Retro } \\
\text { VOC Pre }\end{array}$ & $\begin{array}{l}\text { VOC } \\
\text { Post }\end{array}$ & $\begin{array}{l}\text { VOC 1- } \\
\text { mth FU }\end{array}$ & $\begin{array}{l}\text { VOC 6- } \\
\text { mth FU }\end{array}$ \\
\hline N & \multirow{6}{*}{$\begin{array}{l}\text { EMDR therapy VB2T as } \\
\text { a VCP }\end{array}$} & 24 & 24 & 22 & 20 & 24 & 24 & 22 & 19 \\
\hline Mean & & 7.75 & 0.167 & 0.545 & 0.350 & 2.00 & 6.96 & 6.86 & 6.89 \\
\hline Median & & 8.00 & 0.00 & 0.00 & 0.00 & 2.00 & 7.00 & 7.00 & 7 \\
\hline $\begin{array}{l}\text { Standard } \\
\text { deviation }\end{array}$ & & 1.39 & 0.482 & 0.739 & 0.587 & 0.780 & 0.204 & 0.351 & 0.315 \\
\hline Minimum & & 5 & 0 & 0 & 0 & 1 & 6 & 6 & 6 \\
\hline Maximum & & 10 & 2 & 2 & 2 & 3 & 7 & 7 & 7 \\
\hline
\end{tabular}

Table 4

Descriptives - Memory Vividness (MV), Memory Emotionality (ME), Memory Intensity (MI): pre, post, 1-mth, \& 6-mth follow-up

\begin{tabular}{|llllllllllllll|}
\hline & Intervention & $\begin{array}{l}\text { MV } \\
\text { Pre }\end{array}$ & $\begin{array}{l}\text { MV } \\
\text { Post }\end{array}$ & $\begin{array}{l}\text { MV } \\
\begin{array}{l}\text { mth } \\
\text { FU }\end{array}\end{array}$ & $\begin{array}{l}\text { MV } \\
\text { mth } \\
\text { FU }\end{array}$ & $\begin{array}{l}\text { ME } \\
\text { Pre }\end{array}$ & $\begin{array}{l}\text { ME } \\
\text { Post }\end{array}$ & $\begin{array}{l}\text { ME 1- } \\
\text { mth } \\
\text { FU }\end{array}$ & $\begin{array}{l}\text { ME 6- } \\
\text { mth } \\
\text { FU }\end{array}$ & $\begin{array}{l}\text { MI } \\
\text { Pre }\end{array}$ & $\begin{array}{l}\text { MI } \\
\text { Post }\end{array}$ & $\begin{array}{l}\text { MI 1- } \\
\text { mth } \\
\text { FU }\end{array}$ & $\begin{array}{l}\text { MI 6- } \\
\text { mth } \\
\text { FU }\end{array}$ \\
\hline N & VB2T & 24 & 24 & 22 & 19 & 24 & 24 & 22 & 19 & 24 & 24 & 22 & 19 \\
\hline Mean & 8.04 & 1.42 & 0.227 & 0.579 & 8.33 & 0.417 & 0.0909 & 0.211 & 8.46 & 0.0417 & 0.409 & 0.474 \\
\hline $\begin{array}{l}\text { Standard } \\
\text { deviation }\end{array}$ & 8.00 & 0.00 & 0.00 & 0 & 8.00 & 0.00 & 0.00 & 0 & 8.00 & 0.00 & 0.00 & 0 \\
\hline Minimum & 1.78 & 2.41 & 1.82 & 1.46 & 1.43 & 0.717 & 2.20 & 1.23 & 1.41 & 0.999 & 1.99 & 1.68 \\
\hline Maximum & 5 & 0 & -7 & -3 & 6 & 0 & -8 & -4 & 6 & -4 & -6 & -6 \\
\hline
\end{tabular}

\section{Results}

Of the N=24 EMDR Europe Consultants who took part in the study, all completed the VB2T treatment session and post-treatment, 1-mth, and 6-mth follow-up measures. There were no dropouts from the study. Tables 3 and 4 highlight the descriptive data regarding various measures - SUD, VOC, MV, ME, and MI, and pre, post, 1-mth, and 6-mth follow-up.

\section{Hypothesis 1}

Figure 1 shows the decrease in SUD and the increase in the Validity of Cognition (VOC) at the post, 1- and 6-month follow-up.

Additionally, in more detail, Table 5 highlights the mean, standard deviation, skewness, kurtosis, baseline, and p values for the SUD and VOC at pre, post, 1-mth and 6-mth follow-up and the maintenance of the VB2T treatment effect. 
Table 5

Means, SD, skewness, and kurtosis for SUD and VOC at pre, post, 1-mth \& 6-mth FU

\begin{tabular}{|llllll|}
\hline & Mean (SD) & Skewness (SE) & Kurtosis (SE) & B (SE) & p value \\
\hline Pre-SUD & $7.75(1.39)$ & $-.15(.47)$ & $-.95(.92)$ & 0 & \\
\hline Post-SUD & $.21(.49)$ & $2.72(.47)$ & $7.73(.92)$ & $-1.02(.03)$ & $<.001^{*}$ \\
\hline 1 m FU SUD & $.64(.79)$ & $.78(.49)$ & $-.89(.95)$ & $-.94(.05)$ & $<.001^{*}$ \\
\hline 6m FU SUD & $.23(.95)$ & $-1.74(.51)$ & $6.99(.99)$ & $-.99(.01)$ & $<.001^{*}$ \\
\hline Pre-VOC & $2.02(.79)$ & $-.08(.47)$ & $-1.36(.92)$ & 0 & \\
\hline Post-VOC & $6.96(.20)$ & $-4.90(.47)$ & $24.00(.92)$ & $1.22(.07)$ & $<.001^{*}$ \\
\hline 1 m FU VOC & $6.87(.31)$ & $-2.60(.49)$ & $5.63(.95)$ & $1.21(.08)$ & $<.001^{*}$ \\
\hline 6 m FU VOC & $6.92(.25)$ & $-3.34(.52)$ & $11.19(1.01)$ & $1.22(.08)$ & $<.001^{*}$ \\
\hline
\end{tabular}

There was a substantial reduction in SUD after receiving EMDR in comparison with the baseline assessment $(B=-1.02, S E=.03, p<$ 0.001). The decrease in SUD maintained in the follow up assessments -1 month $(B=-.94, S E=.05, p<0.001)$ and 6 months $(B=-.99$, $S E$ $=.04, p<0.001)$ in comparison with the baseline. Simultaneously, there was an increase in VOC after receiving EMDR in comparison with the baseline assessment $(B=1.22, S E=.07, p<0.001)$. The increase maintained after 1-month $(B=1.21, S E=.08, p<0.001)$ and 6 months $(B=1.22, S E=.08, p<0.001)$ post intervention.

The results of this study indicate that VB2T decreased the SUD and increase the VOC in the treatment of a pathogenic memory tested at pre, post, 1-mth and 6-mth follow-up, suggesting that using VB2T as a video conferencing psychotherapy demonstrated a treatment effect on the pathogenic memory when measured by the SUD and VOC. Furthermore, there was a statistically significant difference between pre-treatment $(M=7.75, S D=1.39)$ and 6-month follow-up $(M=0.35, S D=0.59)$, with a Hedges' $g$ effect size value $(g=6.71)$ suggesting high practical significance. Therefore, we reject the null hypothesis that there is no difference on the Subjective Unit of Disturbance (SUD) or Validity of Cognition (VOC) when using the VB2T EMDR intervention as a video-conference psychotherapy.

\section{Hypothesis 2}

Figure 2 shows the reduction in the nature and characteristics of the pathogenic memory, including memory vividness, emotionality, and intensity. For some research participants, alterations in memory characteristics indicated positive change rather than disturbance (negative), and therefore positive change is presented as a minus score.

As figure 2 demonstrates the VB2T intervention clearly impacted on three areas of distinctiveness of the pathogenic - memory vividness, emotionality, and intensity, with results maintained at both 1-mth and 6-mth FU. There were significant decreases in MV $(B=-.42, S E=.05, p<0.001), M E(B=-.61, S E=.03, p<0.001)$, and $M I(B=-.77, S E=.04, p<0.001)$ following the intervention. These effects maintained in the first month follow-up for $M E(B=-.65, S E=.06, p<0.001), M I(B=-.79, S E=.03, p<0.001), M V(B=-.60, S E=.07$, $p<0.001)$, and sustained after six months for $M V(B=-.58, S E=.03, p<0.001), M E(B=-.62, S E=.03, p<0.001), M I(B=-.79, S E=.04, p<$ $0.001)$. Overall, these results indicate changes that were consistently, statistically significant at $p<0.001$. Additionally, results demonstrate a favourable dose effect, with potential evidence in support of resilience and post-traumatic growth, as indicated the treatment effect emphasises between pre and 6-mth FU. This represents a significant finding from this study.

Therefore, it would be reasonable to assume that when VB2T delivered as a VCP, it has the potential to instigate distinct changes to core components of the pathogenic memory, suggesting evidence of memory reconsolidation. Regarding hypothesis 2: Distinctiveness VB2T, as a VCP, will have no impact in reducing Memory Intensity (MI), Memory Emotionality (ME), and Memory Vividness (MV) of a pathogenic memory following intervention when measured at post-treatment, 1-mth and 6-mth in comparison with a pre-measure, results do not support this, therefore we reject the null hypothesis

\section{Hypothesis 3}

Figures 3 \& 4 demonstrate exposure to Adverse Childhood Experiences (ACEs) or Benevolent Childhood Experiences (BCEs) did not influence the processing of the pathogenic memory or the intervention outcome following the utilisation of VB2T. 
As explained previously, testing the relevance hypothesis compared the study participant group with the original primary studies (35-39, 42). A single factor ANOVA explored the between-group variances. This one-way analysis of variance is a technique used to compare two or more samples when utilising numerical or categorical data.

Figure 4 highlights the prevalence of ACEs between studies $(35,42)$. A descriptive review of the results suggests higher exposure to $4+$ ACEs within the VB2T participant group; however, results yielded a $F(3,4)=8.45$, $p$-value $<.03^{\star}$ suggesting that there were indeed differences between the three groups in terms of the prevalence of ACEs, therefore rejecting the null hypothesis.

Table 6

Types of Adverse Childhood Experiences (ACE) * $\mathrm{P}<.001$

\begin{tabular}{|lll|}
\hline ACEs & Incidence & Sig. (2-test) \\
\hline Psychological abuse & $7(33 \%)$ & .189 \\
\hline Physical abuse & $2(10 \%)$ & $<.001^{\star}$ \\
\hline Sexual abuse & $4(19 \%)$ & .007 \\
\hline Emotional neglect & $9(43 \%)$ & .664 \\
\hline Physical neglect & $2(9 \%)$ & $<.001$ * \\
\hline Parental divorce & $6(26 \%)$ & .035 \\
\hline Mother physical abuse & $0(0 \%)$ & $<.001^{\star}$ \\
\hline Household substance abuse & $4(17 \%)$ & .003 \\
\hline Household mental illness & $8(35 \%)$ & .210 \\
\hline Criminal behaviour in household & $0(0 \%)$ & $<.001$ * \\
\hline
\end{tabular}

Table 6 provides more descriptive data of the VB2T participant group relating to specific exposure to adverse childhood experiences (ACEs). Results focussed on exposure to physical abuse, physical neglect, mother physical abuse, and criminal behaviour in the household.

Although an ANOVA revealed a distinction between the three groups, it is essential to highlight the elevated incidence of exposure to psychological abuse, emotional neglect, household mental illness, absence of exposure to mother physical abuse, and criminal behaviour in the household VB2T research participant group. Figure 5 rank scores the ten questions of the original ACEs questionnaire from most prevalent (1) to least (10).

Figure 6 compares the frequency of both ACEs and BCEs from the VB2T research population. The mean ACE of 1.8 (SD 1.68) and BCE of 7.6 (SD 2.06). The correlation between ACEs \& BCEs is $r(22)=-.48$.

For the benevolent childhood experiences (BCEs), the mean for the sample was $7.6(S D=2.06)$, and the median was 8 . As can be seen from Table 7, most participants reported having eight (17.4\%), nine (21.7\%), and ten (21.7\%) benevolent experiences in their childhood. All participants reported at least four BCEs. Table 5 displays the frequency of each type of BCEs in the current sample. Results suggest that having "At least one good friend" (96\%) and "Opportunities to have a good time" (91\%) were the most frequent benevolent experiences. 
Types of Benevolent Childhood Experiences (BCEs)

\begin{tabular}{|c|c|c|}
\hline BCES & Incidence & Sig. (2-test) \\
\hline At least one caregiver with whom you felt safe & $18(78 \%)$ & .011 \\
\hline At least one good friend & $22(96 \%)$ & $<.001^{\star}$ \\
\hline Beliefs that gave you comfort & $16(70 \%)$ & .093 \\
\hline Enjoyment at school & $18(78 \%)$ & .011 \\
\hline At least one teacher that cared & $20(87 \%)$ & $<.001^{*}$ \\
\hline Good neighbours & $17(74 \%)$ & .035 \\
\hline An adult (not a parent/ caregiver or the person from *11) who could provide you with support or advice & $12(52 \%)$ & 1.000 \\
\hline Opportunities to have a good time & $21(91 \%)$ & $<.001^{*}$ \\
\hline Like yourself or feel comfortable with yourself & $12(52 \%)$ & 1.000 \\
\hline Predictable home routine, like regular meals and a regular bedtime & $20(87 \%)$ & $<.001^{\star}$ \\
\hline
\end{tabular}

In exploring further hypothesis 3: Relevance - firstly, results seem to indicate that neither ACEs nor BCEs score bore no influence on the outcome. did not influence the outcome of the intervention. Secondly, although $75 \%$ of the research participants admitted their motivation for participation being based firmly on 'non-disclosure', however, results indicated $87.5 \%$ did disclose their target memory to the treating clinician. Of these $87.5 \%$, Table 8 highlights the categories of target memories disclosed.

Table 8

Disclosed target memory themes and frequencies chosen by research participants for VB2T as a VCP

o sexual assault (3)
o child abuse (4)
o parental neglect (1)
o fatal road traffic collision (1)
o occupational bullying (4)
o complicated grief (2)
o episodes involving shame and humiliation (6)

As indicated earlier, using VB2T demonstrates a distinct treatment effect with Table 8 highlighting clinical applicability. In addition, the desensitisation and reprocessing of these trauma memories occurred irrespective of either ACEs or BCEs. These results were consistent at 1 and 6-month FU. Consequently, this data set supports the assertion of hypothesis 3.

Regarding hypothesis 3 results suggest rejection of the null in relation to ACEs and BCEs.

In testing hypothesis 4: Efficiency - the administration of VB2T was tested against the period recommended by EMDRIA sessions; 60 90 minutes. Results for this study used a time metric (minutes) from commencement of Phase 3 - Assessment, to completion of Phase 7 - Closure (including debrief). Of the $\mathrm{N}=24$ research participants, the average VB2T session was 57 minutes and 27 seconds, with an SD of 17 minutes 27 seconds. Results highlight that the treatment sessions were below the 60 Minutes threshold.

Testing the costing element within hypothesis 4 required economic modelling using University of Worcester financial algorithms. The UW costing model used for each VB2T treatment session was calculated at $£ 56.49$ ( $€ 66.36$ ). Of the 24 clinical sessions of VB2T carried out the mean cost per session was $£ 54.02$ ( $€ 63.45$ ). This represented potential modest savings of $£ 2.47$ ( $€ 2.91$ ) per session. However, Figure 7 indicates the variance in treatment costs of each individual session. An additional consideration one of the distinct advantages of remote intervention is the reduction in client related costs, such as travel time, transportation, parking, etc. 
Results indicate savings in terms of time and efficiency, with additional health economic benefits. Regarding hypothesis 4 results suggest rejection of the null.

\section{Discussion}

The rationale for this study was to ascertain how EMDR therapy could be used as a video conferencing psychotherapy considering the current Covid-19 pandemic where social distancing is a vital strategy in reducing infection rates. However, more specifically, this research wanted to explore the potential use of the EMDR therapy Blind 2 Therapist Protocol (VB2T) virtual version -as a VCP-to determine its fitness for purpose, distinctiveness, and relevance and efficiency.

The research participants for this study represented highly experienced EMDR therapy clinicians, all of whom were actively engaged in current clinical practice during the Covid-19 pandemic. Despite being experienced clinicians, many of the research participants had not previously encountered VCP EMDR therapy in the role of 'client'. One participant disclosed that they had not received EMDR therapy since their initial training - which was 20 years previously. The fact that the average SUD score for the N-24 research participants was 7.75 (SD 1.39) is worthy of note. The memory targets identified in Table 6 suggest that the research participants worked on pathogenic memories of major adverse life events; the mean SUD score, nonetheless, is intriguing. An expectation would suggest a higher mean SUD considering the trauma themes involved. This would certainly account for such an extremely large effect size $\left(\right.$ hedges $\left.^{\prime} g=6.71\right)$. This issue is worthy of further investigation and consideration.

Caution does need to be exercised with regards to hypothesis 1. Results indicate an extremely large effect size, although alterations in SUD and VOC are highly consistent in EMDR therapy clinical practice, the studies research population are experienced EMDR therapists

- highly conversant with EMDR therapy practice, and therefore already extremely positive towards EMDR therapy. For this reason, future research needs to utilise both an experimental design and a clinical population, both of which would potentially deliver a more realistic treatment effect size.

To the best of our knowledge, this is the first EMDR therapy study that has examined both Adverse (ACEs) and Benevolent (BCEs) childhood experiences. The most significant ACEs was emotional neglect, exposure to household mental illness, and psychological abuse up to 18 years of age. However, exposure to mother physical abuse, and criminal behaviour in the household, with exposure to physical abuse and physical neglect, presented as the more prevalent ACEs in this research population.

The BCEs highlighted positive early life experiences in adults that built resilience and provided a counterbalance to ACEs. These positive childhood experiences include effective caregiving, quality in parenting, close relationships with other significant adults, effective schooling, and community. Higher BCEs is associated with more favourable long-term development (108). Within the participant group, the most decisive factors included having at least one good friend, at least one teacher that cared, opportunities to have a good time, and a predictable home routine. Although the data set indicates high levels of BCEs, caution is required as the study participants were highly trained mental health professionals and are not a clinical population. That considered, other interesting observable aspects from the dataset highlight a dichotomy between having an adult (other than a caregiver) who could provide support and advice and liking or feeling comfortable with oneself. More research is needed to understand further the impact that ACEs \& BCEs have on and explore further if and how BCEs act as a potential resilience to counteract the impact of ACEs. However, the results of this study highlight that trauma processing occurred using VB2T irrespective of the research participants ACE or BCE scores.

Regarding testing hypotheses 1 through 4, the results from the study indicate a treatment effect from using the remotEMDR software to carry out VB2T as a Video-conferencing Psychotherapy (VCP). Although the alterations in SUD and VOC are highly consistent with the more comprehensive empirical support for EMDR therapy, the data highlighting personal changes to the characteristics of the pathogenic memory targeted from processing is undoubtedly intriguing. Again, caution is necessary as the sample size is relatively small and would need further testing with a clear clinical population with a more formal medico-legal diagnosis. Nonetheless, results are auspicious.

In testing hypothesis 3 - relevance, results for disclosure (90\%) appear consistent with the previous B2T study in Northern Iraq (18) highlighting a clear clinical benefit in using both B2T \& VB2T EMDR therapy protocols. This VB2T study adds to the literature demonstrating equal effectiveness and suggests a potential correlation between non-disclosure and level of SUD. The desensitisation and reprocessing of the pathogenic memory increase the probability of disclosure with results of $87.5 \%$ for this VB2T study. The clinical advantages of this make this an effective tool to use as a trauma treatment intervention, highlighting a distinct benefit of using EMDR

Page $11 / 22$ 
therapy for undisclosed trauma memories compared to other trauma-focussed interventions. Again, caution is required as further research is needed to investigate this aspect.

The use of three additional subjective measure - memory intensity, memory vividness, and memory emotionality, within this study suggests an argument for including these within the EMDR therapy B2T and the VB2T protocol, but also within the standard protocol. These measures appear particularly useful in understanding the subjective experience of the trauma memory targeted for processing. Further research and investigation are needed to pursue this argument further.

Although this was only a 1-treatment session study, the data set reveals an interesting health economic argument with an average session cost of $£ 56.49$ ( $€ 66.36$ ). Although the study yielded modest economic savings of $£ 2.47$ ( $€ 2.91$ ) per session the cumulative implications of this, in addition with the clinical benefits, suggest a particularly compelling argument.

There are two major flaws within this study. Firstly, the research participant group - they are not on a non-clinical population, they are also highly positive in disposition towards EMDR therapy. Moving towards a study with a distinct clinical population is essential for further research. Secondly, this was a straightforward pre-test - post-test research design, a future study would need to utilise a more experimental framework. As the study involves non-disclosure of a trauma memory, randomisation would not be possible, for ethical reasons, and therefore a quasi-experimental design could be a viable alternative.

To summarise, the research results suggest VB2T EMDR therapy to be an effective, fit for purpose, safe to use trauma treatment intervention. Additionally, results highlight its clinical relevance and applicability as a trauma intervention. Furthermore, the remotEMDR software provided a highly effective platform offering in delivering EMDR therapy as a VCP. Although the researchers acknowledge that other platforms exist, results from this study are based entirely on remotEMDR.

\section{Conclusion}

This research study demonstrated encouraging evidence in support of EMDR therapy as video-conferencing psychotherapy in treating a pathogenic (trauma) memory. In addition, the study explored specific factors influenced by the treatment intervention. What this study's results highlight is the potential in using EMDR therapy, in this case using the B2T protocol as a VCP. Furthermore, results suggest the intervention to have clinical applicability. Caution does need to be exercised regard both the lack of a clinical population, and the need for a more experimental design.

However, to have an intervention that appears effective with either shame, or fear-based trauma memories suggests great potential regarding clinical applicability. To have such an intervention that works on trauma memories clients are unwilling to disclose, for fear, blame or prejudice, suggests distinct advantages for EMDR therapy in the repertoire of empirically supported trauma treatment interventions. Furthermore, to have such an intervention that appears safe and effective adds more temerity to this assertion. This study highlights how the VB2T EMDR therapy scripted protocol alters core characteristics of the pathogenic memory itself, including memory disturbance, emotionality, intensity, and vividness. Results also demonstrate that these changes occur irrespective of either ACEs or BCEs. Another critical finding relates to resilience and post-traumatic growth factors more powerful when considering a stark choice for clients - disclosure of the memory or no treatment, they choose no treatment. To provide a credible alternative in this critical decisionmaking juncture suggests distinct clinical benefits and applicability. Potential health efficiency arguments highlighted by this research are tentative yet worthy of further investigation and critical consideration.

In summary, these results demonstrate proof of concept, and puts forward the case for further research and investigation. The stage 2 aspect of the study will further test the EMDR intervention as a VCP with a defined control group. The research supports the case for EMDR therapy as a credible treatment when used as a video conference psychotherapy. Although a compelling argument this is still a pilot study and not a clinical one, therefore, scalability would require further investigation with a distinct clinical population. At this stage, results from this pilot study need to be viewed with some degree of caution as this stage 1 research incorporated a self-selecting participant group, with an existing, and strong, disposition towards EMDR therapy. This bias needs to be both open and transparent. That said, the Covid-19 pandemic has challenged existing mental health and psychology services enormously. As the global burden of psychological trauma continues unabated, and we remain in an environment of scarcity in resources, any intervention that provides distinct choice and effectiveness in treating shame or feared based memories is a compelling argument, and much needed treatment approach. That such an intervention has potential health efficiency benefits strengthens this argument further. Any intervention, virtual or not, which improves accessibility and reach must be welcomed.

Page $12 / 22$ 


\section{Declarations}

Ethics approval: Ethical approval for the study was granted through University of Worcester (UK) [CBPS1920031-R2]. Consequently, all the methods used for the study were carried out in strict adherence to the ethical approval granted and in accordance with relevant guidelines and regulations.

Ethics Statement: This study involved human participants, and therefore ethical approval for the study was granted by University of Worcester (UK), College of Health, Life, \& Environmental Sciences [CBPS1920031-R2]. The research participants provided their written consent to participate in the study.

Consent: Informed consent was obtained from all subjects

Consent for publication: as the Principal Investigator of the research team, on behalf of the team, we give consent for publication

Data Availability Statement: The raw data supporting this research, and subsequent article, is available from the Open-Source Repository https://osf.io/dashboard

Competing interests: we declare that there are no competing interests with regards to this research project or subsequent publication.

Funding: We declare that this was an unfunded study

Author Contributions: DF conceived the study, caried out the VB2T treatment sessions, and was the Chief Investigator for the project. LK, $\mathrm{PM}$, and ZZ were part of the research team and acquired the follow-up date and conducted the qualitative interviews. AF, DF \& MK carried of the data analysis. The primary author was the principal investigator; however, all authors contributed to the overall article and approved the submitted version for publication.

\section{Conflicts of Interest}

The authors declare that the research was conducted in the absence of any commercial or financial relationship that could be construed as a potential conflict of interest. No incentives, financial or otherwise were offered to research participants.

Although remotEMDR is a commercial product no financial inducements were offered to conduct the research.

\section{References}

1. Turgoose D, Ashwick R, Murphy D. Systematic review of lessons learned from delivering tele-therapy to veterans with post-traumatic stress disorder. Journal of Telemedicine and Telecare. 2018;24(9).

2. Wind TR, Rijkeboer M, Andersson G, Riper H. The COVID-19 pandemic: The 'black swan' for mental health care and a turning point for e-health. Vol. 20, Internet Interventions. 2020.

3. Anderson P, Rothbaum BO, Hodges LF. Virtual Reality Exposure in the Treatment of Social Anxiety. Cognitive and Behavioral Practice. 2003;10(3).

4. Berryhill MB, Halli-Tierney A, Culmer N, Williams N, Betancourt A, King M, et al. Videoconferencing psychological therapy and anxiety: A systematic review. Vol. 36, Family Practice. 2018.

5. Berryhill MB, Culmer N, Williams N, Halli-Tierney A, Betancourt A, Roberts H, et al. Videoconferencing Psychotherapy and Depression: A Systematic Review. Vol. 25, Telemedicine and e-Health. 2019.

6. Germain V, Marchand A, Bouchard S, Guay S, Drouin MS. Assessment of the therapeutic alliance in face-to-face or videoconference treatment for posttraumatic stress disorder. Cyberpsychology, Behavior, and Social Networking. 2010;13(1).

7. Christopher Frueh B, Grubaugh AL, Elhai JD, Buckley TC. US Department of Veterans Affairs Disability Policies for posttraumatic stress disorder: Administrative trends and implications for treatment, rehabilitation, and research. Vol. 97, American Journal of Public Health. 2007.

8. Karyotaki E, Kemmeren L, Riper H, Twisk J, Hoogendoorn A, Kleiboer A, et al. Is self-guided internet-based cognitive behavioural therapy (iCBT) harmful? An individual participant data meta-Analysis. Psychological Medicine. 2018;48(15).

9. Karyotaki E, Ebert DD, Donkin L, Riper H, Twisk J, Burger S, et al. Do guided internet-based interventions result in clinically relevant changes for patients with depression? An individual participant data meta-analysis. Vol. 63, Clinical Psychology Review. 2018.

Page $13 / 22$ 
10. Osenbach JE, O’Brien KM, Mishkind M, Smolenski DJ. Synchronous telehealth technologies in psychotherapy for depression: A meta-analysis. Depression and Anxiety. 2013;30(11).

11. Rousmaniere T, Abbass A, Frederickson J, Henning I, Taubner S. Videoconference for psychotherapy training and supervision: Two case examples. Vol. 68, American Journal of Psychotherapy. 2014.

12. Ruskin PE, Silver-Aylaian M, Kling MA, Reed SA, Bradham DD, Hebel JR, et al. Treatment outcomes in depression: Comparison of remote treatment through telepsychiatry to in-person treatment. American Journal of Psychiatry. 2004;161(8).

13. Tuerk PW, Keller SM, Acierno R. Treatment for Anxiety and Depression via Clinical Videoconferencing: Evidence Base and Barriers to Expanded Access in Practice. FOCUS. 2018;16(4).

14. Watts S, Marchand A, Bouchard S, Gosselin P, Langlois F, Belleville G, et al. Telepsychotherapy for generalized anxiety disorder: Impact on the working alliance. Journal of Psychotherapy Integration. 2020;30(2).

15. Weinberg H. Online group psychotherapy: Challenges and possibilities during COVID-19-A practice review. Vol. 24, Group Dynamics. 2020.

16. Simpson SG, Reid CL. Therapeutic alliance in videoconferencing psychotherapy: A review. Australian Journal of Rural Health. $2014 ; 22(6)$.

17. Crowe M, Inder M, Farmar R, Carlyle D. Delivering psychotherapy by video conference in the time of COVID-19: Some considerations. Journal of Psychiatric and Mental Health Nursing. 2020.

18. Farrell D, Kiernan MD, de Jongh A, Miller PW, Bumke P, Ahmad S, . . \&, et al. Treating implicit trauma: a quasi-experimental study comparing the EMDR Therapy Standard Protocol with a 'Blind 2 Therapist' version within a trauma capacity building project in Northern Iraq. 5, 1-13. Journal of International Humanitarian Action. 2020;1-13.

19. Morland LA, Hynes AK, MacKintosh MA, Resick PA, Chard KM. Group cognitive processing therapy delivered to veterans via telehealth: A pilot cohort. Journal of Traumatic Stress. 2011;24(4).

20. Myers KM, Valentine JM, Melzer SM. Child and adolescent telepsychiatry: Utilization and satisfaction. Telemedicine and e-Health. 2008;14(2).

21. Backhaus A, Agha Z, Maglione ML, Repp A, Ross B, Zuest D, et al. Videoconferencing psychotherapy: A systematic review. Psychological Services. 2012;9(2).

22. Baños RM, Etchemendy E, Mira A, Riva G, Gaggioli A, Botella C. Online positive interventions to promote well-being and resilience in the adolescent population: A narrative review. Frontiers in Psychiatry. 2017;8(JAN).

23. Barak A, Hen L, Boniel-Nissim M, Shapira N. A comprehensive review and a meta-analysis of the effectiveness of internet-based psychotherapeutic interventions. Journal of Technology in Human Services. 2008;26(2-4).

24. Bolton P, Bass JK, Zangana SAS, Kamal T, Murray MMI, Kaysen D, et al. A randomized controlled trial of mental health interventions for survivors of systematic violence in Kurdistan, Northern Iraq. BMC Psychiatry. 2014;14(1).

25. Wild J, Warnock-Parkes E, Grey N, Stott R, Wiedemann M, Canvin L, et al. Internet-delivered cognitive therapy for PTSD: a development pilot series. European Journal of Psychotraumatology. 2016;7(1).

26. Berger T. The therapeutic alliance in internet interventions: A narrative review and suggestions for future research. Psychotherapy Research. 2017;27(5).

27. World Health Organisation. Mental health and psychosocial considerations during the COVID-19 outbreak, 18 March 2020 . WHO 18th March 2020:(No. WHO/2019-nCoV/MentalHealth/2020.1). World Health Organization. 2020.

28. Cullen W, Gulati G, Kelly BD. Mental health in the COVID-19 pandemic. Vol. 113, QJM. 2020.

29. Ghebreyesus TA. Addressing mental health needs: an integral part of COVID-19 response. World Psychiatry. 2020.

30. Kavoor AR. COVID-19 in People with Mental IIIness: Challenges and Vulnerabilities. Vol. 51, Asian Journal of Psychiatry. 2020.

31. Khan KS, Mamun MA, Griffiths MD, Ullah I. The Mental Health Impact of the COVID-19 Pandemic Across Different Cohorts. International Journal of Mental Health and Addiction. 2020.

32. Kumar A, Nayar KR. COVID 19 and its mental health consequences. Journal of Mental Health. 2020.

33. Talevi D, Socci V, Carai M, Carnaghi G, Faleri S, Trebbi E, et al. Mental health outcomes of the covid-19 pandemic. Rivista di Psichiatria. 2020;55(3).

34. Usher K, Durkin J, Bhullar N. The COVID-19 pandemic and mental health impacts. Vol. 29, International Journal of Mental Health Nursing. 2020.

Page $14 / 22$ 
35. Bellis MA, Lowey H, Leckenby N, Hughes K, Harrison D. Adverse childhood experiences: Retrospective study to determine their impact on adult health behaviours and health outcomes in a UK population. Journal of Public Health (United Kingdom). 2014;36(1).

36. Bellis MA, Hughes K, Ford K, Ramos Rodriguez G, Sethi D, Passmore J. Life course health consequences and associated annual costs of adverse childhood experiences across Europe and North America: a systematic review and meta-analysis. The Lancet Public Health. 2019;4(10).

37. Bellis MA, Hughes K, Leckenby N, Hardcastle KA, Perkins C, Lowey H. Measuring mortality and the burden of adult disease associated with adverse childhood experiences in England: A national survey. Vol. 37, Journal of Public Health (United Kingdom). 2015.

38. Bellis MA, Hardcastle K, Ford K, Hughes K, Ashton K, Quigg Z, et al. Does continuous trusted adult support in childhood impart lifecourse resilience against adverse childhood experiences - a retrospective study on adult health-harming behaviours and mental well-being. BMC psychiatry. 2017;17(1).

39. Bellis MA, Hughes K, Leckenby N, Jones L, Baban A, Kachaeva M, et al. Adverse childhood experiences and associations with health-harming behaviours in young adults: Surveys in eight eastern European countries. Bulletin of the World Health Organization. 2014;92(9).

40. Brown DW, Anda RF, Tiemeier H, Felitti VJ, Edwards VJ, Croft JB, et al. Adverse Childhood Experiences and the Risk of Premature Mortality. American Journal of Preventive Medicine. 2009;37(5).

41. Burke NJ, Hellman JL, Scott BG, Weems CF, Carrion VG. The impact of adverse childhood experiences on an urban pediatric population. Child Abuse and Neglect. 2011;35(6).

42. Felitti VJ, Anda RF, Nordenberg D, Williamson DF, Spitz AM, Edwards V, \&, et al. Relationship of childhood abuse and household dysfunction to many of the leading causes of death in adults: The Adverse Childhood Experiences (ACE) Study. American journal of preventive medicine, 14(4), 245-258. 1998;245-58.

43. Islam T, Pitafi AH, Arya V, Wang Y, Akhtar N, Mubarik S, et al. Panic buying in the COVID-19 pandemic: A multi-country examination. Journal of Retailing and Consumer Services. 2021;59.

44. Kim P, Thomas J, Wilk J, Castro C, Hoge C. Stigma, Barriers to Care, and Use of Mental Health Services Among Active Duty and National Guard Soldiers After Combat. Psychiatric Services. 2010;61(6).

45. Acierno R, KR, TP, GAK, LC, RK, . . \& \&EB. A non-inferiority trial of prolonged exposure for posttraumatic stress disorder: in person versus home-based telehealth. . Behaviour Research and Therapy. 2017;57-65.

46. Acierno R, GDF, RKJ, HMA, KRG, LCW, . . \& TPW. Behavioral activation and therapeutic exposure for posttraumatic stress disorder: A noninferiority trial of treatment delivered in person versus home-based telehealth. . Depression \& Anxiety. 2016;415-23.

47. Clapp JD, Kemp JJ, Cox KS, Tuerk PW. Patterns of change in response to Prolonged Exposure: Implications for treatment outcome. Depression and Anxiety. 2016;33(9).

48. Hernandez-Tejada MA, Zoller JS, Ruggiero KJ, Kazley AS, Acierno R. Early treatment withdrawal from evidence-based psychotherapy for PTSD: Telemedicine and in-person parameters. International Journal of Psychiatry in Medicine. 2014;48(1).

49. Strachan M, Gros DF, Yuen E, Ruggiero KJ, Foa EB, Acierno R. Home-based telehealth to deliver evidence-based psychotherapy in veterans with PTSD. Contemporary Clinical Trials. 2012;33(2).

50. Yuen EK, GDF, PM, ZS, TPW, FEB, \& AR (2015). Randomized controlled trial of home-based telehealth versus in-person prolonged exposure for combat-related PTSD in veterans: Preliminary results. Journal of Clinical Psychology. 2015;500-12.

51. Luxton DD, PLD, OK, \& KG. An evaluation of the feasibility and safety of a home-based telemental health treatment for posttraumatic stress in the US military. Telemedicine and e-Health. 2015;880-6.

52. Fortney JC, Pyne JM, Kimbrell TA, Hudson TJ, Robinson DE, Schneider R, et al. Telemedicine-based collaborative care for posttraumatic stress disorder: A randomized clinical trial. JAMA Psychiatry. 2015;72(1).

53. Grubbs KM, Fortney JC, Pyne JM, Hudson T, Moore WM, Custer P, et al. Predictors of Initiation and Engagement of Cognitive Processing Therapy Among Veterans With PTSD Enrolled in Collaborative Care. Journal of Traumatic Stress. 2015;28(6).

54. Maieritsch KP, Smith TL, Hessinger JD, Ahearn EP, Eickhoff JC, Zhao Q. Randomized controlled equivalence trial comparing videoconference and in person delivery of cognitive processing therapy for PTSD. Journal of Telemedicine and Telecare. 2016;22(4).

55. Olthuis J v., Watt MC, Bailey K, Hayden JA, Stewart SH. Therapist-supported Internet cognitive behavioural therapy for anxiety disorders in adults. The Cochrane database of systematic reviews. 2016;3.

Page 15/22 
56. Olthuis J v., Wozney L, Asmundson GJG, Cramm H, Lingley-Pottie P, McGrath PJ. Distance-delivered interventions for PTSD: A systematic review and meta-analysis. Vol. 44, Journal of Anxiety Disorders. 2016.

57. Lightstone AJ, Bailey SK, Voros P. Collaborative music therapy via remote video technology to reduce a veteran's symptoms of severe, chronic PTSD. Arts and Health. 2015;7(2).

58. Todder D, Kapln Z. Rapid eye movements for acute stress disorder using video conference communication. Telemedicine and eHealth. 2007;13(4).

59. Todder D, Matar M, Kaplan Z. Acute-phase trauma intervention using a videoconference link circumvents compromised access to expert trauma care. Telemedicine Journal and e-Health. 2007;13(1).

60. Bongaerts H, Voorendonk EM, van Minnen A, de Jongh A. Safety and effectiveness of intensive treatment for complex PTSD delivered via home-based telehealth. European Journal of Psychotraumatology. 2021;12(1).

61. Bisson JI, Berliner L, Cloitre M, Forbes D, Jensen TK, Lewis C, et al. The International Society for Traumatic Stress Studies New Guidelines for the Prevention and Treatment of Posttraumatic Stress Disorder: Methodology and Development Process. Journal of Traumatic Stress. 2019;32(4).

62. American Psychological Society. Clinical Practice Guideliness for the Treatment of PTSD. APA. 2017;

63. ISTSS Posttraumatic Stress Disorder Prevention and Treatment Guidelines Methodology and Recommendations. ISTSS. 2018

64. Karatzias T, Shevlin M, Fyvie C, Grandison G, Garozi M, Latham E, et al. Adverse and benevolent childhood experiences in Posttraumatic Stress Disorder (PTSD) and Complex PTSD (CPTSD): implications for trauma-focused therapies. European Journal of Psychotraumatology. 2020;11(1).

65. Karatzias T, Murphy P, Cloitre M, Bisson J, Roberts N, Shevlin M, et al. Psychological interventions for ICD-11 complex PTSD symptoms: systematic review and meta-analysis. Psychological Medicine. 2019 Aug 12;49(11).

66. de Jongh A, Bicanic I, Matthijssen S, Amann BL, Hofmann A, Farrell D, et al. The Current Status of EMDR Therapy Involving the Treatment of Complex Posttraumatic Stress Disorder. Journal of EMDR Practice and Research. 2019;13(4).

67. Hase M, Balmaceda UM, Ostacoli L, Liebermann P, Hofmann A. The AIP model of EMDR therapy and pathogenic memories. Frontiers in Psychology. 2017;8(SEP).

68. Valiente-Gómez A, Moreno-Alcázar A, Treen D, Cedrón C, Colom F, Pérez V, et al. EMDR beyond PTSD: A systematic literature review. Vol. 8, Frontiers in Psychology. 2017.

69. Shapiro. Eye movement desensitization and reprocessing (EMDR) therapy. Basic Principles, Protocols, and Procedures, Ed, 3. New York: Guilford Press; 2018.

70. de Jongh A, Amann BL, Hofmann A, Farrell D, Lee CW. The Status of EMDR Therapy in the Treatment of Posttraumatic Stress Disorder 30 Years After Its Introduction. Journal of EMDR Practice and Research. 2019;13(4).

71. de Jongh A, Groenland GN, Sanches S, Bongaerts H, Voorendonk EM, van Minnen A. The impact of brief intensive trauma-focused treatment for PTSD on symptoms of borderline personality disorder. European Journal of Psychotraumatology. 2020;11(1).

72. Matthijssen SJMA, Brouwers TC, van den Hout MA, Klugkist IG, de Jongh A. A randomized controlled dismantling study of Visual Schema Displacement Therapy (VSDT) vs an abbreviated EMDR protocol vs a non-active control condition in individuals with disturbing memories. European Journal of Psychotraumatology. 2021;12(1).

73. Bennett-Levy J, Lee NK. Self-practice and self-reflection in cognitive behaviour therapy training: What Factors Influence Trainees' Engagement and Experience of Benefit? Behavioural and Cognitive Psychotherapy. 2014;42(1).

74. Chigwedere C, Bennett-Levy J, Fitzmaurice B, Donohoe G. Personal practice in counselling and CBT trainees: the self-perceived impact of personal therapy and self-practice/self-reflection on personal and professional development. Cognitive Behaviour Therapy. 2020

75. Collard J, \& CM. Experiential learning for trainee therapists through a shame attack exercise. The Cognitive Behavioural Therapist. 2020;13-undefined.

76. Scott J, Yap K, Bunch K, Haarhoff B, Perry H, Bennett-Levy J. Should personal practice be part of cognitive behaviour therapy training? Results from two self-practice/self-reflection cohort control pilot studies. Clinical Psychology and Psychotherapy. $2021 ; 28(1)$.

77. Wolpe J, \& LAA. Behavior therapy techniques: A guide to the treatment of neuroses. 1966.

78. Shapiro F. Eye movement desensitization and reprocessing: Basic principles, protocols, and procedures. New York: Guilford Press. 1995. 
79. Shapiro F. Eye movement desensitization and reprocessing: Basic principles, protocols, and procedures (2nd ed.). New York: Guilford Press; 2001.

80. Andrade J, Kavanagh D, Baddeley A. Eye-movements, and visual imagery: A working memory approach to the treatment of posttraumatic stress disorder. British Journal of Clinical Psychology. 1997;36(2).

81. Engelhard IM, van den Hout MA, Janssen WC, van der Beek J. Eye movements reduce vividness and emotionality of "flashforwards." Behaviour Research and Therapy. 2010;48(5).

82. Gunter RW, Bodner GE. How eye movements affect unpleasant memories: Support for a working-memory account. Behaviour Research and Therapy. 2008;46(8).

83. Leer A, \& EIM. Side effects of induced lateral eye movements during aversive ideation. Journal of behavior therapy and experimental psychiatry. 2020

84. Schubert SJ, Lee CW, Drummond PD. Eye movements matter, but why? Psychophysiological correlates of EMDR therapy to treat trauma in Timor-Leste. Journal of EMDR Practice and Research. 2016;10(2).

85. Schubert SJ, Lee CW, Drummond PD. The efficacy and psychophysiological correlates of dual-attention tasks in eye movement desensitization and reprocessing (EMDR). Journal of Anxiety Disorders. 2011;25(1).

86. van den Hout MA, Engelhard IM, Beetsma D, Slofstra C, Hornsveld H, Houtveen J, et al. EMDR and mindfulness. Eye movements and attentional breathing tax working memory and reduce vividness and emotionality of aversive ideation. Journal of Behavior Therapy and Experimental Psychiatry. 2011;42(4).

87. Maxfield L, Melnyk WT, Hayman GCA. A Working Memory Explanation for the Effects of Eye Movements in EMDR. Journal of EMDR Practice and Research. 2008 Nov;2(4).

88. van der Kolk BA. The body keeps the score: Brain, mind, and body in the healing of trauma. Penguin Books.; 2015.

89. van der Kolk BA. Psychological trauma. American Psychiatric Pub. American Psychiatric Pub.; 2003.

90. van der Kolk BA, SJ, BME, HJW, HEK, KDL, \& SWB. A randomized clinical trial of eye movement desensitization and reprocessing (EMDR), fluoxetine, and pill placebo in the treatment of posttraumatic stress disorder: Treatment effects and long-term maintenance. Journal of Clinical Psychiatry. 2007;68(1):37-46.

91. Afifi TO, Taillieu T, Salmon S, Davila IG, Stewart-Tufescu A, Fortier J, et al. Adverse childhood experiences (ACEs), peer victimization, and substance use among adolescents. Child abuse \& neglect. 2020;106.

92. Aronson KR, Perkins DF, Morgan NR, Bleser JA, Vogt D, Copeland LA, et al. The impact of adverse childhood experiences (ACEs) and combat exposure on mental health conditions among new post-9/11 veterans. Psychological Trauma: Theory, Research, Practice, and Policy. 2020;12(7).

93. Felitti VJ, \& Anda RF. The relationship of Adverse Childhood Experiences to adult medical disease, psychiatric disorders, and sexual behavior: Implications for healthcare. In R. Lanius \& E Vermetten (Eds.). The hidden epidemic: The impact of early life trauma on health and disease. Cambridge University Press, New York. 2010;77-87.

94. Finkelhor D. Trends in Adverse Childhood Experiences (ACEs) in the United States. Child Abuse and Neglect. 2020;108.

95. Narayan AJ, Ippen CG, Harris WW, Lieberman AF. ASSESSING ANGELS IN THE NURSERY: A PILOT STUDY OF CHILDHOOD MEMORIES OF BENEVOLENT CAREGIVING AS PROTECTIVE INFLUENCES. Infant Mental Health Journal. 2017;38(4).

96. Struck S S-TAAAAGAT. Adverse childhood experiences (ACEs) research: A bibliometric analysis of publication trends over the first 20 years. . Child Abuse \& Neglect. 2021 Feb;1;112:104895.

97. Crandall A BESMMBNMHCBMD. The influence of adverse and advantageous childhood experiences during adolescence on young adult health. Child Abuse \& Neglect 2020 Oct . 2020;1;108:104644.

98. Crandall A MJCANLGRNMMBLBBMHCL. ACEs and counter-ACEs: How positive and negative childhood experiences influence adult health. . Child abuse \& neglect. 2019 Oct;96.

99. Doom JR, Seok D, Narayan AJ, Fox KR. Adverse and Benevolent Childhood Experiences Predict Mental Health During the COVID-19 Pandemic. Adversity and Resilience Science. 2021;

100. Malti T. Kindness: a perspective from developmental psychology. European Journal of Developmental Psychology. 2020.

101. Merrick JS, Narayan AJ. Assessment and screening of positive childhood experiences along with childhood adversity in research, practice, and policy. Journal of Children and Poverty. 2020;26(2).

102. Merrick JS, Narayan AJ, DePasquale CE, Masten AS. Benevolent Childhood Experiences (BCEs) in homeless parents: A validation and replication study. Journal of Family Psychology. 2019;33(4). 
103. Starbird AD, Story PA. Consequences of childhood memories: Narcissism, malevolent, and benevolent childhood experiences. Child Abuse and Neglect. 2020;108.

104. Farrell D, Reid K. EMDR Therapy with a Head and Neck Cancer Client Group. In: Eye Movement Desensitization and Reprocessing (EMDR) Therapy Scripted Protocols and Summary Sheets. 2020.

105. Maxfield L, SNJ, \& SR. EMDR Fidelity Rating Scale (EFRS). EMDR Research Foundation. 2018

106. Locascio JJ, Atri A. An Overview of Longitudinal Data Analysis Methods for Neurological Research. Dementia and Geriatric Cognitive Disorders Extra. 2011;1(1).

107. Locascio JJ, \& AA. An overview of longitudinal data analysis methods for neurological research. Dementia and geriatric cognitive disorders extra. 2011;330-57.

108. Masten AS. Global Perspectives on Resilience in Children and Youth. Child Development. 2014;85(1).

\section{Figures}

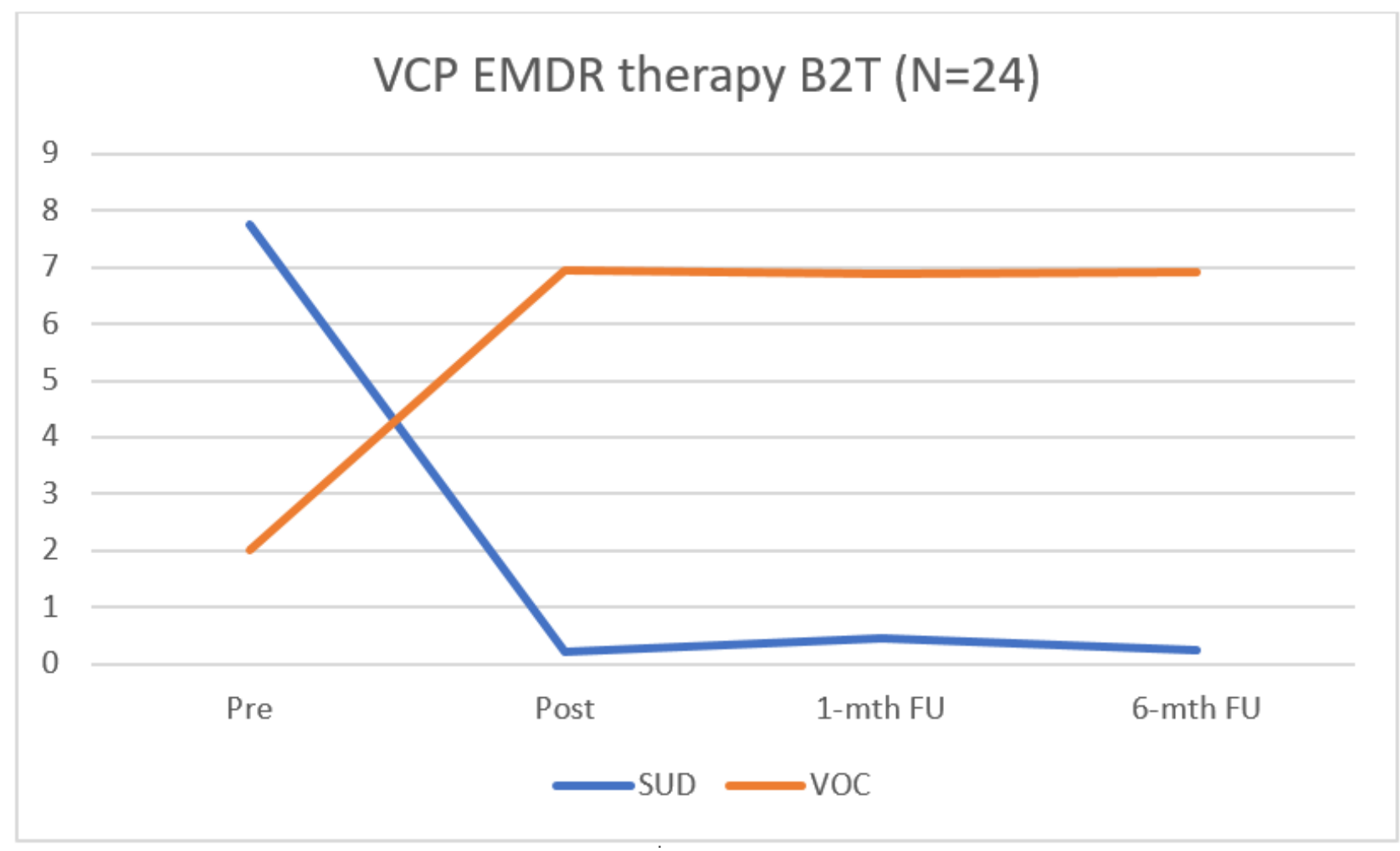

\section{Figure 1}

Alterations in SUD \& VOC scores at pre, post, 1-mth \& 6-mth FU 


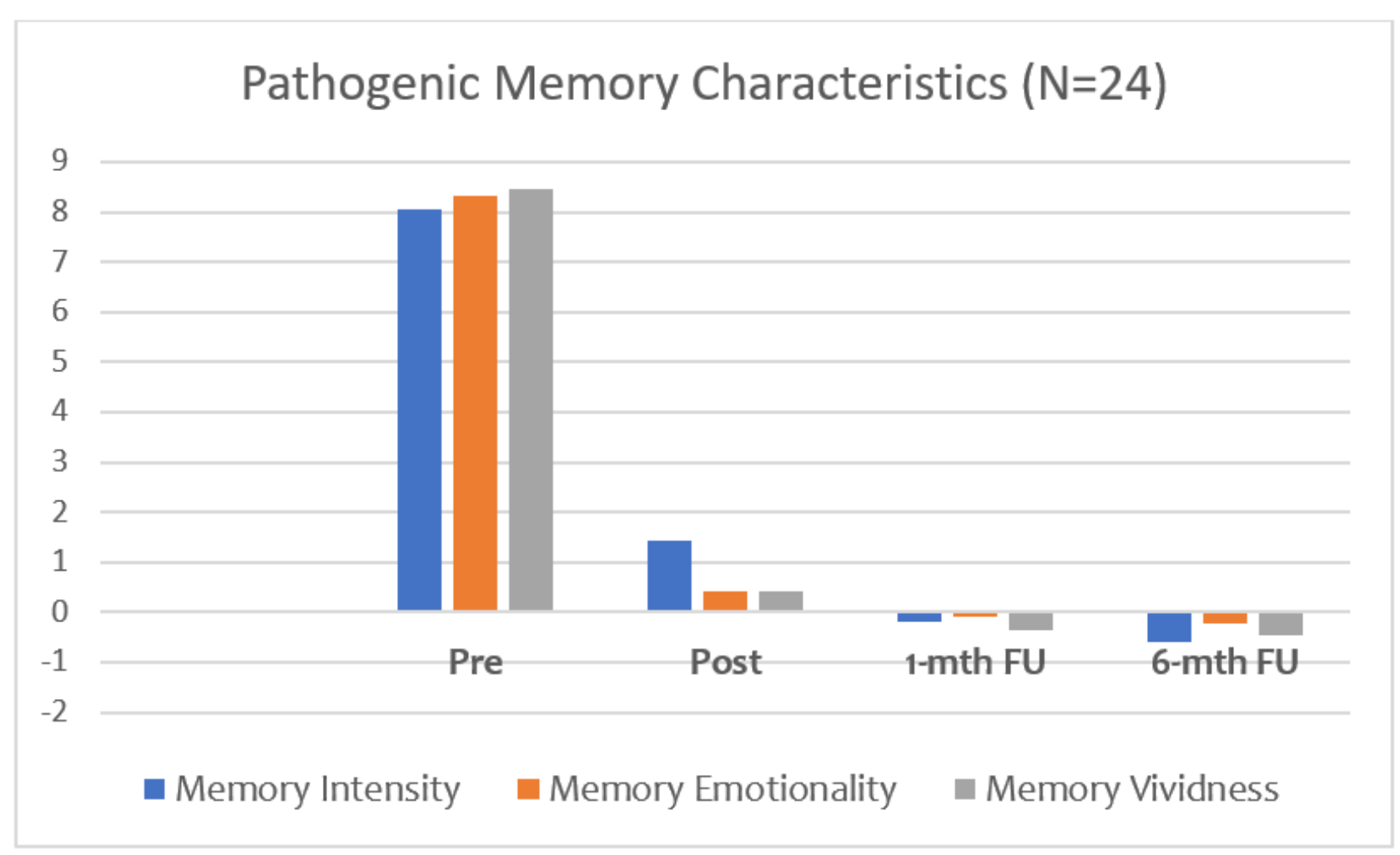

Figure 2

Changes in Pathogenic Memory Subjective Characteristics

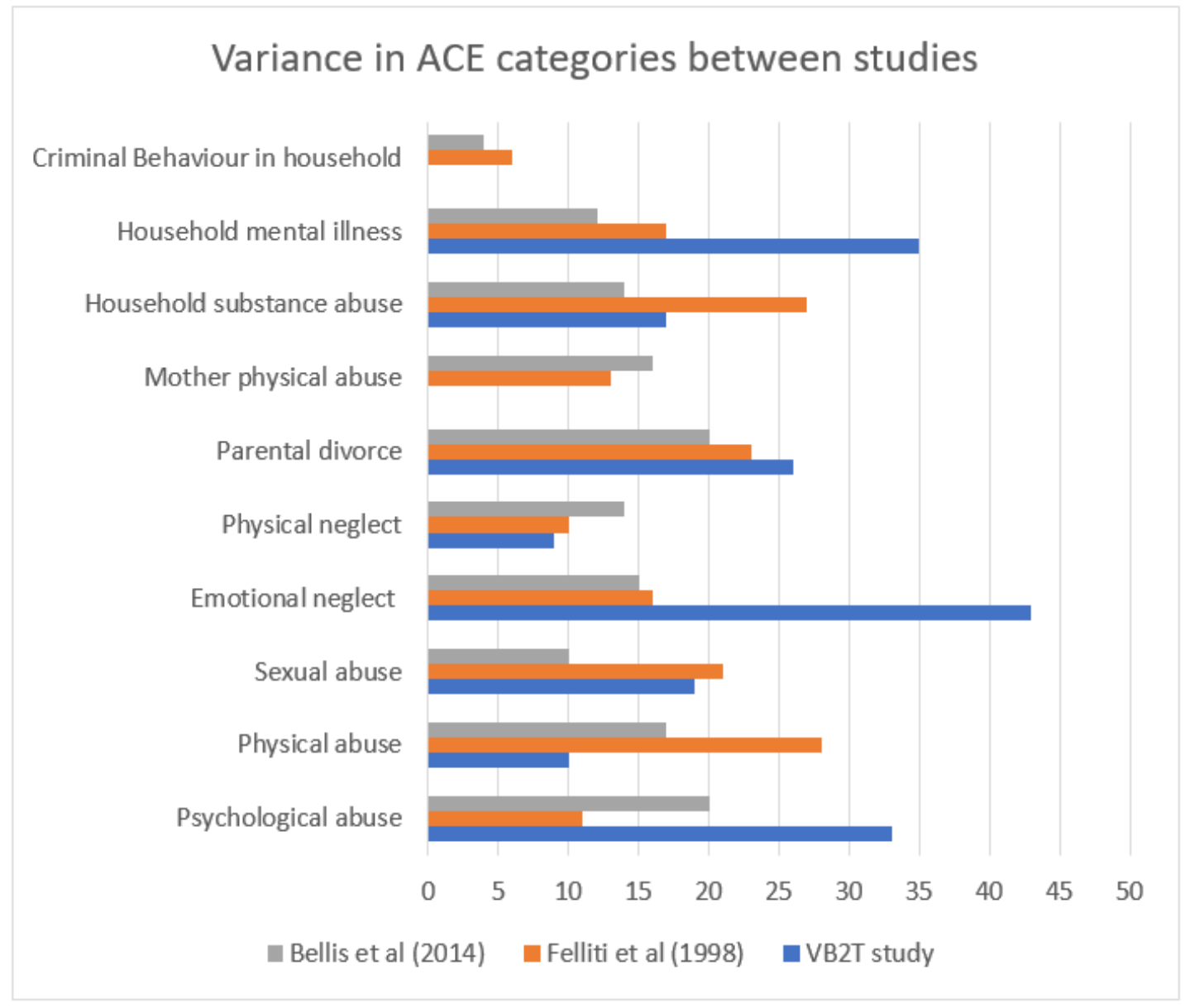

Figure 3 


\section{Prevalence of ACEs between studies}

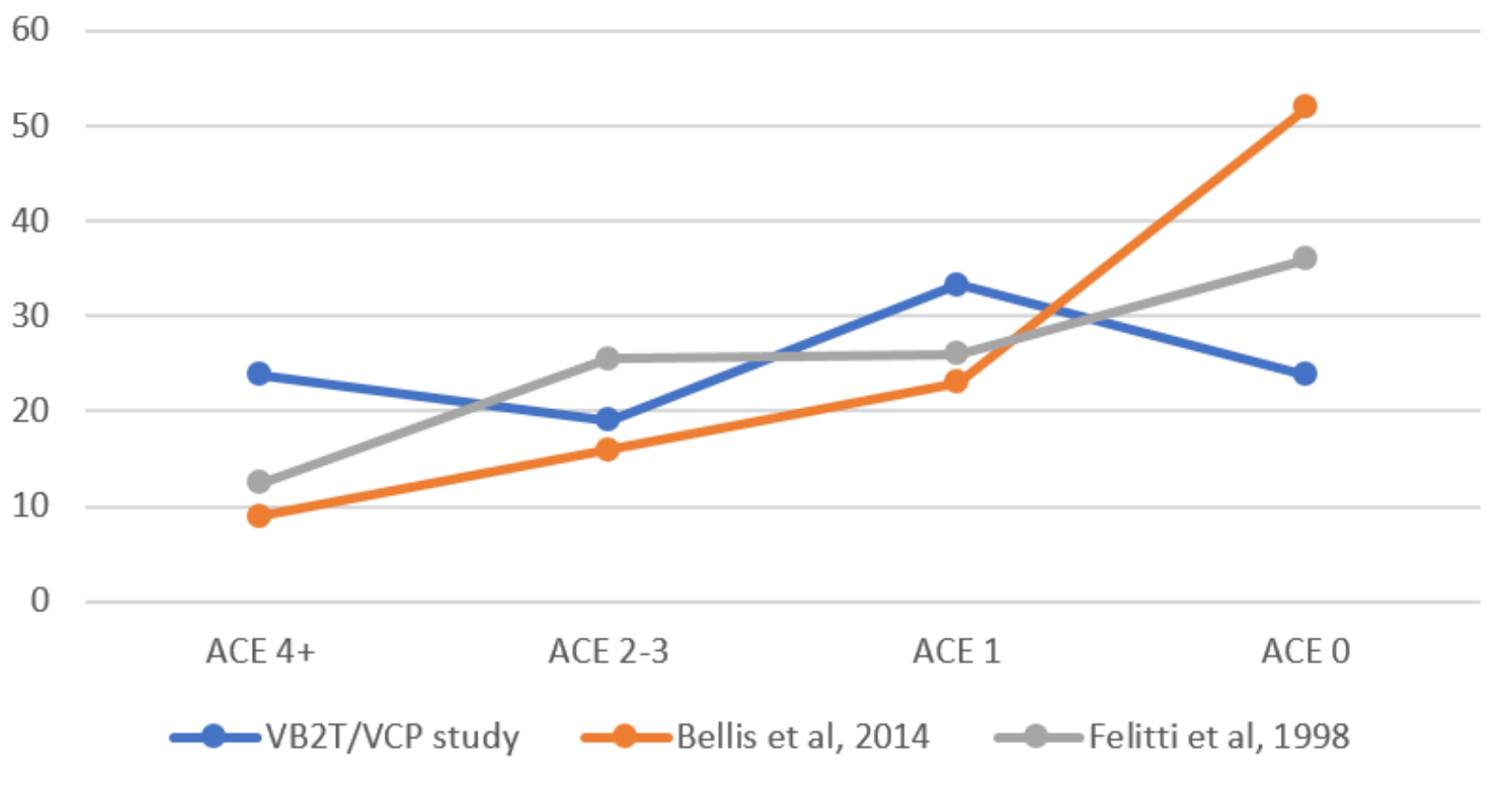

\section{Figure 4}

Prevalence of ACE scores between the three groups: VB

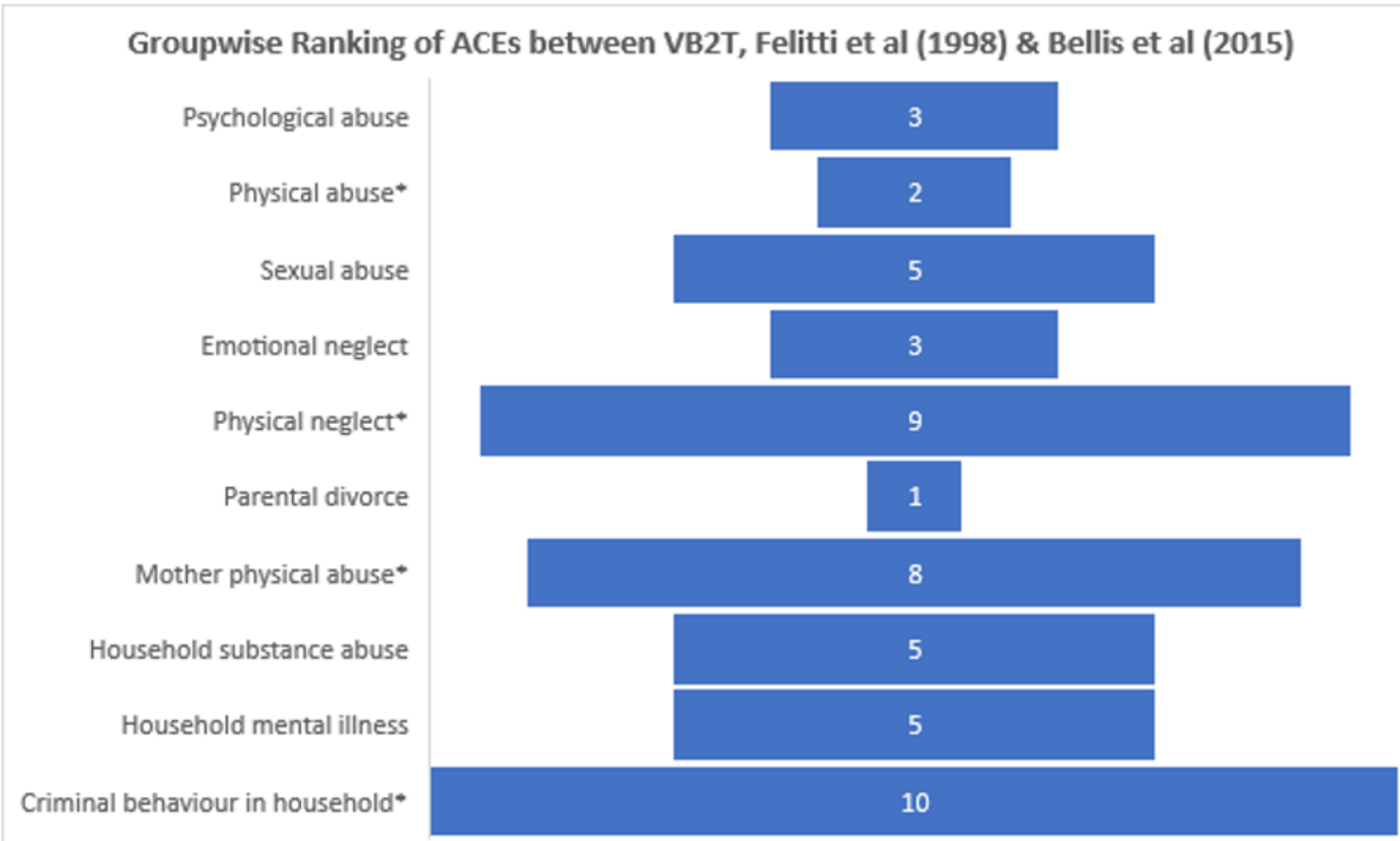

Figure 5 


\section{ACE's \& BCE's scores ( $\mathrm{N}=23)$}

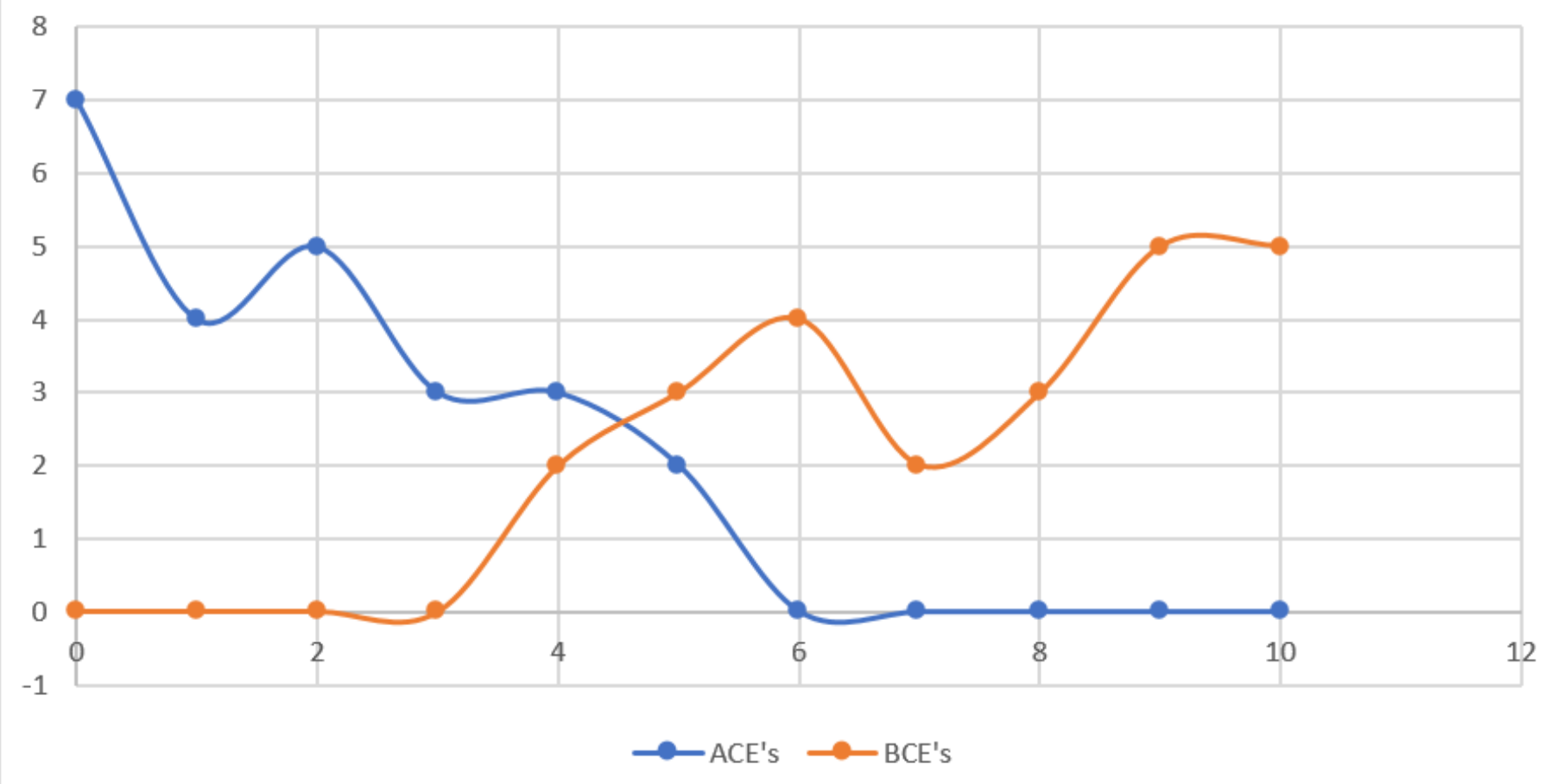

\section{Figure 6}

Frequency of Adverse (ACE's) \& Benevolent (BCE's) scores of the research participants

\section{Cost per session $\mathrm{f}^{\prime} \mathrm{s}$ for VB2T $(\mathrm{N}=\mathbf{2 4})$}

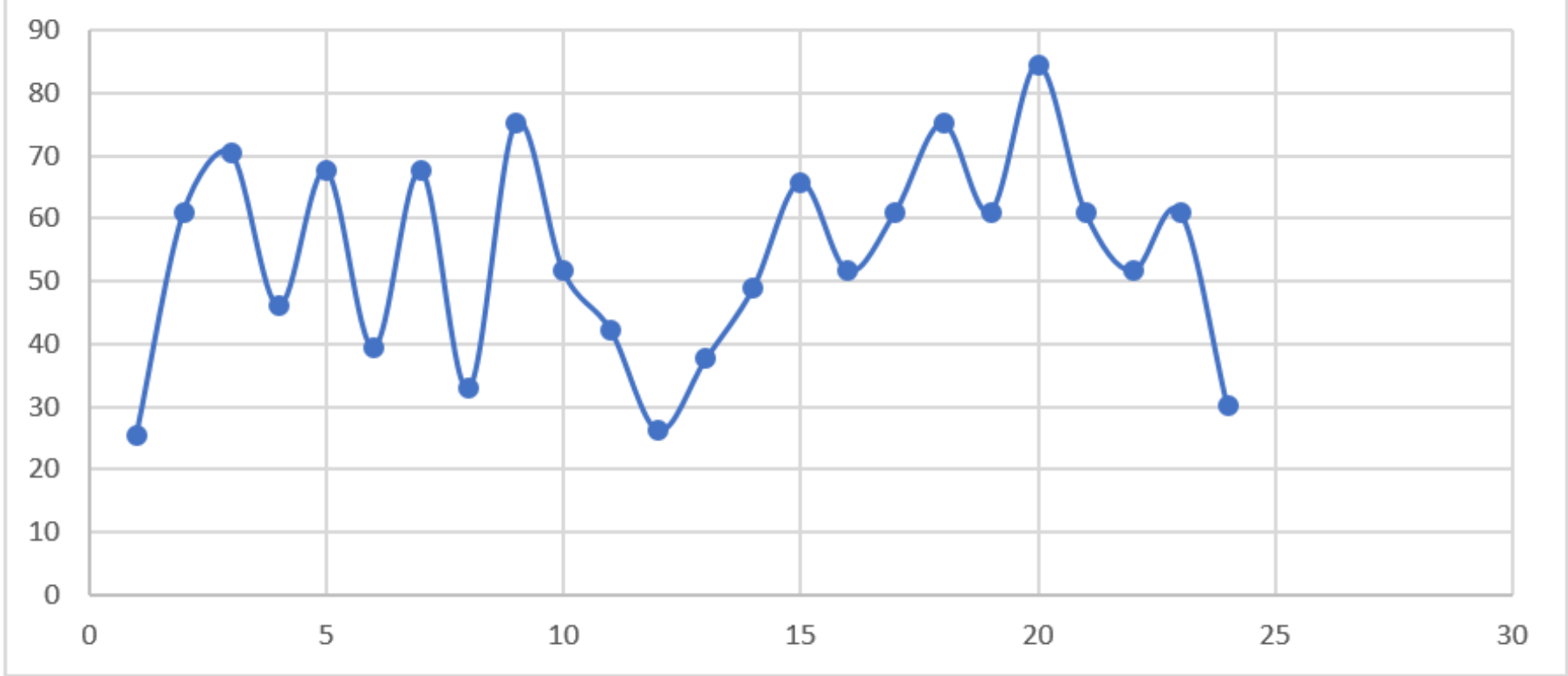

\section{Figure 7}

Variance in cost of VB2T treatment sessions carried out as a VCP

\section{Supplementary Files}


This is a list of supplementary files associated with this preprint. Click to download.

- EMDRasVCPResearchProtocolDF18102021.docx

- CONSORT2010Checklist.doc 